\title{
DISCOVERY OF A POSSIBLE COOL WHITE DWARF COMPANION FROM THE AIIWISE MOTION SURVEY
}

\author{
Sergio B. Fajardo-Acosta ${ }^{1}$, J. Davy Kirkpatrick ${ }^{1}$, Adam C. Schneider ${ }^{2}$, Michael C. Cushing $^{2}$, Daniel Stern ${ }^{3}$, \\ Christopher R. Gelino ${ }^{1}$, Daniella C. Bardalez-Gagliuffi ${ }^{4}$, Kendra KellogG ${ }^{5}$, and Edward L. Wright ${ }^{6}$ \\ ${ }^{1}$ IPAC, Mail Code 100-22, Caltech, 1200 E. California Boulevard, Pasadena, CA 91125, USA; \\ fajardo@ipac.caltech.edu, davy@ipac.caltech.edu,cgelino@ipac.caltech.edu \\ ${ }^{2}$ University of Toledo, 2801 W. Bancroft Street, MS 113, Toledo, OH 43606, USA; aschneid10@gmail.com, michael.cushing@utoledo.edu \\ ${ }^{3}$ Jet Propulsion Laboratory, California Institute of Technology, 4800 Oak Grove Drive, MS 169-221, Pasadena, CA 91109, USA; daniel.k.stern@jpl.nasa.gov \\ ${ }^{4}$ University of California at San Diego, 9450 Gillman Drive \# 40282, La Jolla, CA 92092, USA; daniella@physics.ucsd.edu \\ ${ }^{5}$ Western University, 226-376-3530, 454 Castlegrove Boulevard, London, ON N6G 1K8, Canada; kkellog@uwo.ca \\ ${ }^{6}$ University of California at Los Angeles, Department of Physics and Astronomy, P.O. Box 951547, Los Angeles, CA 90095-1547, USA; wright@astro.ucla.edu \\ Received 2016 April 22; revised 2016 September 16; accepted 2016 September 17; published 2016 November 17
}

\begin{abstract}
We present optical and near-infrared spectroscopy of WISEA J061543.91-124726.8, which we rediscovered as a high motion object in the AllWISE survey. The spectra of this object are unusual; while the red optical $(\lambda>7000 \AA)$ and near-infrared spectra exhibit characteristic TiO, VO, and $\mathrm{H}_{2} \mathrm{O}$ bands of a late-M dwarf, the blue portion of its optical spectrum shows a significant excess of emission relative to late-M-type templates. The excess emission is relatively featureless, with the exception of a prominent and very broad $\mathrm{Na}$ I D doublet. We find that no single, ordinary star can reproduce these spectral characteristics. The most likely explanation is an unresolved binary system of an M7 dwarf and a cool white dwarf. The flux of a cool white dwarf drops in the optical red and near-infrared, due to collision-induced absorption, thus allowing the flux of a late-M dwarf to show through. This scenario, however, does not explain the Na D feature, which is unlike that of any known white dwarf, but which could perhaps be explained via unusual abundance or pressure conditions.
\end{abstract}

Key words: brown dwarfs - solar neighborhood - stars: individual (WISEA 0615-1247) - stars: low-mass subdwarfs - white dwarfs

\section{INTRODUCTION}

The study and census of our Solar neighborhood is important to advance our understanding of low-mass stars and brown dwarfs, which are the most numerous known objects in the Galaxy. As such, they shed light on the low-end of the initial mass function, and on star formation efficiency. The search for our nearest neighbors lets us study objects in great detail, because they are the closest, brightest objects of their class. The detailed study of late-type dwarfs, brown dwarfs, subdwarfs, and white dwarfs has historically relied on the identification of objects based on their optical through mid-infrared colors. These studies have been enhanced by recent surveys for high proper motion objects, because these surveys have no color bias, and can therefore identify objects with unusual characteristics not found through color-based searches.

The object 2MASS J06154357-1247221 = WISEA J061543.91-124726.8, which we henceforth designate WISEA 0615-1247, was originally discovered as a high proper motion star by Lépine (2008) and designated by him as PM I06157 -1247 in a re-analysis of southern hemisphere digitized sky surveys. Lépine (2008) finds proper motion components for

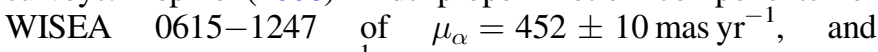
$\mu_{\delta}=-421 \pm 10$ mas yr $^{-1}$ from measurements spanning an epoch range of 39.2 years (see Table 1). The object was rediscovered by Kirkpatrick et al. (2014) due to its high motion in the AllWISE Data Release of the Wide-Field Infrared Survey Explorer (WISE; Wright et al. 2010). The colors of the object, namely $J-K_{s}=0.937 \pm 0.058 \mathrm{mag}$, from the Two Micron All-Sky Survey (2MASS; Skrutskie 1997), and $J-W 2=$ $1.685 \pm 0.040 \mathrm{mag}$ (from 2MASS $J$ and the WISE $4.6 \mu \mathrm{m}$ bandpass $W 2$; see Table 1), are shown as the red symbol in Figure 1. This figure compares these colors with those of 47,936 high motion sources (Kirkpatrick et al. 2014, 2016). WISEA
0615-1247 lies $\sim 0.2$ mag below or blueward in $J-K_{s}$ color relative to the normal sequence of field objects, possibly hinting at a cool subdwarf nature. Early-L subdwarfs have colors $J-W 2$ ranging from 1.1 to $1.8 \mathrm{mag}$, and $J-K_{s}$ on average 0.23 mag below the normal sequence of field objects (see Figure 7 in Kirkpatrick et al. 2016). The suggested sub-dwarf classification of the object is similar to that by Lépine (2008), based on the reduced proper motion of the object in the $V$ bandpass. In order to elucidate the nature of the object, we obtained optical and near-infrared spectroscopy. The spectrum, as discussed below, proved to be unique. We propose a binary scenario that we believe best explains the observed data, although it invokes a cool white dwarf companion unlike any heretofore cataloged.

\section{OBSERVATIONS}

\subsection{Proper Motion}

The AllWISE Source Catalog ${ }^{7}$ lists R.A. Motion $=593 \pm$ $73 \mathrm{mas} \mathrm{yr}^{-1}$ and decl. Motion $=-322 \pm 78 \mathrm{mas} \mathrm{yr}^{-1}$ for WISEA 0615-1247 (Table 1). In order to confirm this motion, we measured the displacement of the object between the 2MASS and AllWISE positions, to yield $\mu_{\alpha}=436.9 \pm$ 6.4 mas yr$^{-1}$ and $\mu_{\delta}=-406.8 \pm 6.5$ mas yr$^{-1}$ (Table 1). This proper motion is in rough agreement, within the errors, with the AllWISE motions. We carried out an additional estimate of the motion of the object, via a $\chi^{2}$ minimization fit to its positions in

\footnotetext{
See http://wise2.ipac.caltech.edu/docs/release/allwise/expsup/. Motions listed in the AllWISE source Catalog are not strictly proper motions, because parallax effects are not taken into account. Hence we list them as "R.A. Motion" and "decl. Motion," to differentiate them from proper motions from other catalogs, which we list as " $\mu_{\alpha}$ " and " $\mu_{\delta}$."
} 
Table 1

General Observational Data of WISEA 0615-1247

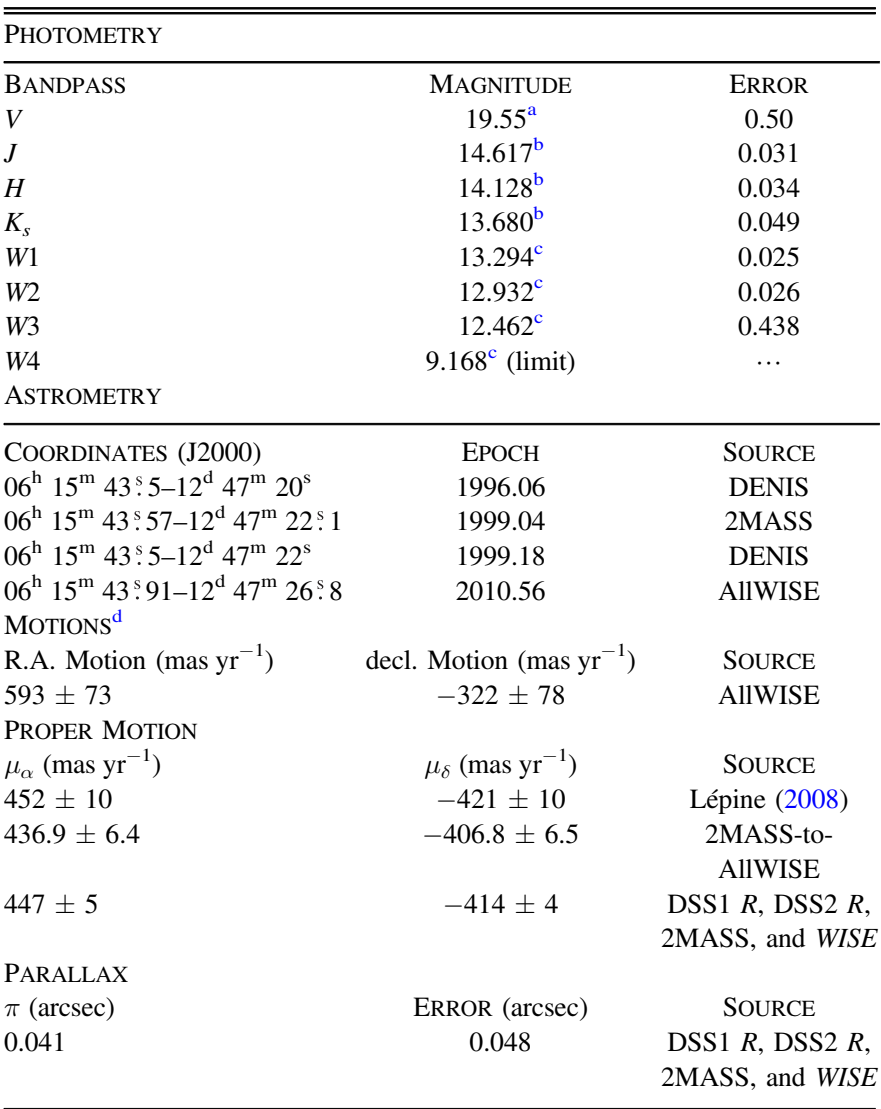

Notes.

${ }^{a}$ From Lépine (2008), estimated from photographic magnitudes using the method of Lépine \& Shara (2005).

${ }^{\mathrm{b}}$ From 2MASS.

${ }^{c}$ From AllWISE.

${ }^{\mathrm{d}}$ Motions from AllWISE are not strictly proper motions; see the AllWISE Explanatory Supplement at http://wise2.ipac.caltech.edu/docs/release/ allwise/expsup/.

the First and Second Digitized Sky Surveys (DSS1 and DSS2) ${ }^{8}$ red ( $R$-band) plates, 2MASS, WISE All-Sky ${ }^{9}$, and WISE 3Band Cryo ${ }^{10}$ Data Releases. In this way, we obtained

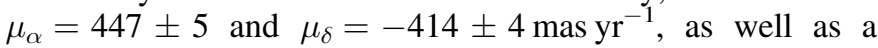
parallax $\pi$, which is practically 0 due to the large errors listed in Table 1. The proper motion of the object thus dominates the AllWISE measured motion.

\subsection{Optical Spectroscopy}

We observed the object with the Double Spectrograph (DBSP; Oke \& Gunn 1982) on the Hale 200 inch Telescope at Palomar Observatory, on UT 2014 February 23, September 27, October 24, and November 15. On the blue side of DBSP, a grating with 600 lines $/ \mathrm{mm}$ blazed at $4000 \AA$ was used for all nights. On the red side, a grating with 600 lines/ $\mathrm{mm}$ blazed at $10000 \AA$ was used, except on November 15 ,

\footnotetext{
8 See http://archive.stsci.edu/dss/.

9 See http://wise2.ipac.caltech.edu/docs/release/allsky/expsup/.

${ }^{10}$ See http://wise2.ipac.caltech.edu/docs/release/allsky/expsup/section7_ 1.html.
}

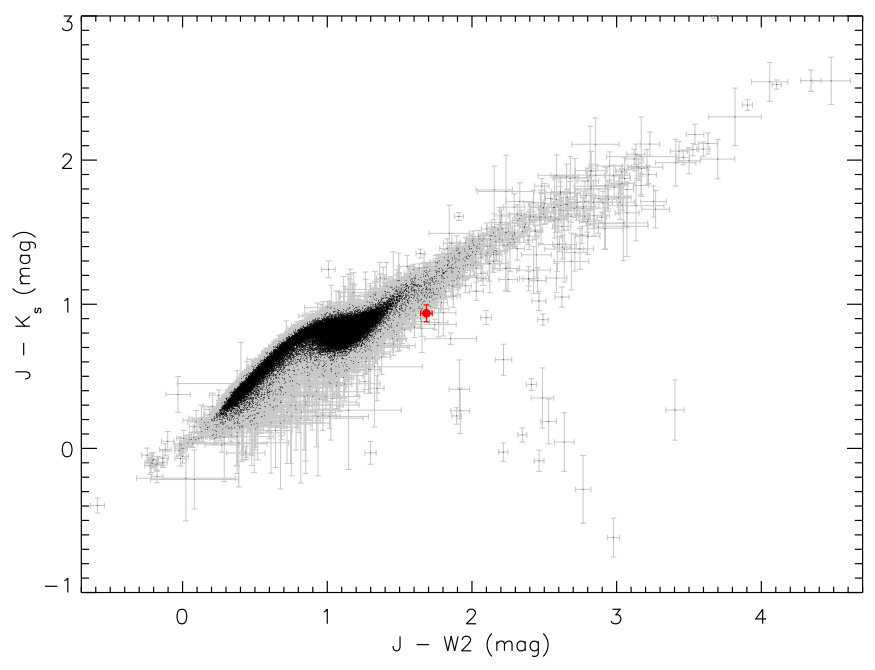

Figure 1. Color $J-K_{s}$ plotted as a function of color $J-W 2$ for 47,936 high motion objects (black symbols with gray error bars) from Kirkpatrick et al. $(2014,2016)$. The red filled circle marks the location of WISEA 0615-1247.

when a red side grating with 316 lines/mm blazed at $7500 \AA$ was used. The D68 dichroic, which splits the light between the blue and red sides near $6800 \AA$, was used on most nights, except on November 15, when the D55 dichroic was used, which splits the light near $5500 \AA$. The resulting spectra covered the range of 4100-9900 $\AA$, except that the spectrum on November 15 covered from 2900 to $10640 \AA$. Grating angles were selected for each dichroic in order to provide wavelength overlap between the blue and red sides of the spectra. Typical spectral resolution with the 1.0 arcsec slit was $\sim 3.6 \AA$ in the blue, and $\sim 2.4 \AA$ in the red. The integration times with DBSP ranged from $3360 \mathrm{~s}$ on October 24 to $6060 \mathrm{~s}$ on November 15. Conditions were clear on February 23; windy with thin clouds on September 27; clear with 0". 8 seeing on October 24; and some cloudiness on November 15.

We also observed the object with the Low Resolution Imaging Spectrometer (LRIS; Oke et al. 1995) on the Keck I $10 \mathrm{~m}$ telescope at the W. M. Keck Observatory, on UT 2014 October 20. On the blue side of LRIS, a grating with 600 lines/ $\mathrm{mm}$ blazed at $4000 \AA$ was used, and on the red side a grating with 400 lines $/ \mathrm{mm}$ blazed at $8500 \AA$ was used. The 560 dichroic was used to split light between the blue and red sides, near $5600 \AA$. The resulting spectrum covered the range from 3300 to $10000 \AA$ A. The spectral resolution with the 1.0 arcsec slit was $\sim 4.6 \AA$ in the blue, and $\sim 8.2 \AA$ in the red. The integration time with LRIS was $600 \mathrm{~s}$, and there were some clouds as remnants from hurricane Ana. Reductions for DBSP observations made use of standard procedures for CCD data, described in Kirkpatrick et al. (2016). Reductions for the LRIS observation made use of a non-stardard procedure, because weather conditions prevented the observation of a flux calibrator on that night. Instead, a flux calibrator (the white dwarf HZ 44) was measured on UT 2015 July 17, using a different instrument configuration than for the target. Therefore, we had to force the spectral shape of the LRIS data of the target at $8590-10270 \AA$ to match that of the DBSP 2014 October 24 data, by fitting second order polynomials. Figure 2 shows all measured optical spectra of WISEA 0615-1247.

We obtained optical spectroscopy of the M7 dwarf G59-32B, also designated WISEA $124007.18+204828.9$, noted as a 


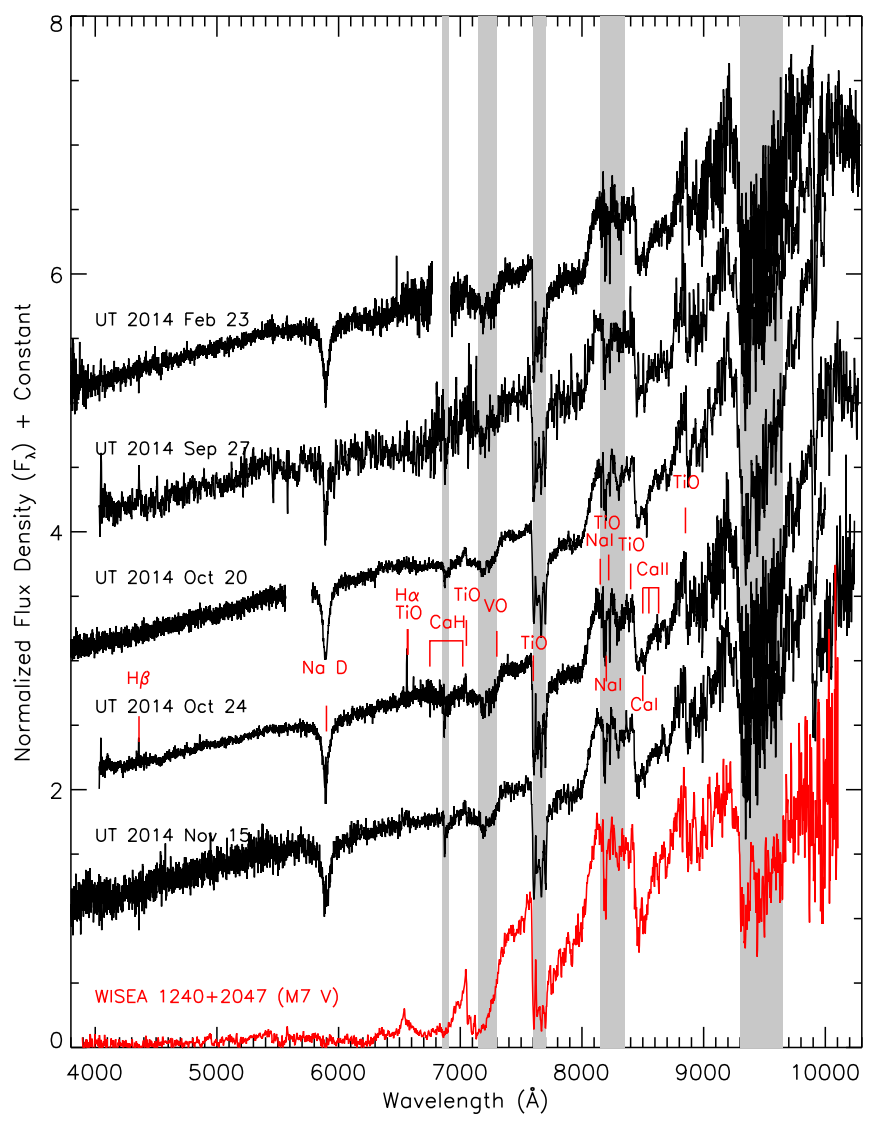

Figure 2. Optical spectra of WISEA 0615-1247, measured with DBSP at Palomar Observatory, on UT 2014 February 23, September 27, October 24, and November 15, and with LRIS at Keck Observatory, on UT 2014 October 20. The bottom spectrum, shown in red, is of the M7 dwarf G59-32B, or WISEA 124007.18+204828.9, for spectral-type comparison. Spectra have been normalized at $7500 \AA$, and a constant offset has been added to separate the spectra vertically. Regions of low atmospheric transmission (Hamuy et al. 1994) are indicated by gray bands. Feature identifications are shown in red for the October 24 spectrum of WISEA 0615-1247.

component in a common-proper motion binary by Kirkpatrick et al. (2014). The system was in a pre-flare state during the observations, and was intended as a spectral-type comparison. We used the Boller \& Chivens Spectrograph on the Irenee du Pont $2.5 \mathrm{~m}$ telescope at Las Campanas Observatory, on UT 2014 May 4. The integration time was 1800 s. See Kirkpatrick et al. (2016) for more details.

We also obtained DBSP observations of three white dwarfs for comparison with WISEA 0615-1247, and another object that was initially believed to also be a white dwarf. The white dwarfs LHS 3250 and LSPM J1702+7158N were observed on UT 2015 June 8, with $3660 \mathrm{~s}$ integrations each, under clear conditions but with $2^{\prime \prime}$ seeing. The white dwarf WD J0205 -053 was observed on UT 2015 September 7, with $2400 \mathrm{~s}$ integrations, through some clouds and glow from a last-quarter moon, although with 1!"1-1!"2 seeing. The 600 lines $/ \mathrm{mm}$ gratings on the blue and red sides of DBSP were again used with the D68 dichroic, to produce continuous 4100-9900 spectra. Details on these three white dwarfs are given in Section 3.2.1. We observed the object LP 343-35 = WISEA $221515.51+315731.9$ (Kirkpatrick et al. 2016), under the assumption that is was the white dwarf WD $2213+317$. This DBSP observation was on UT 2016 January 13, with a $300 \mathrm{~s}$

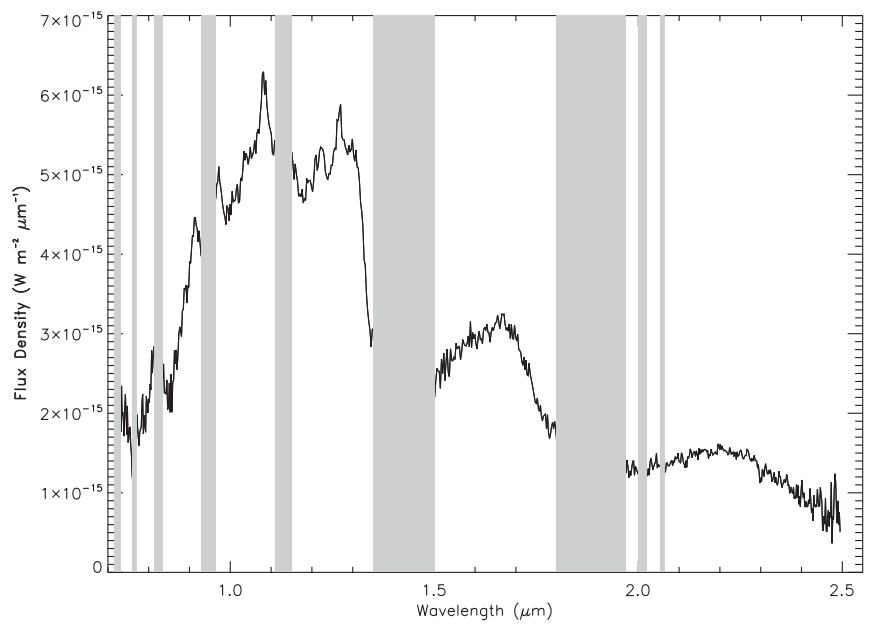

Figure 3. Near-infrared spectrum of WISEA 0615-1247, measured with the SpeX spectrograph at the NASA IRTF, on UT 2015 January 18. Regions of telluric absorption (Rayner et al. 2009) are indicated by gray bands.

integration, using the D55 dichroic, and a $1.5 \mathrm{arcsec}$ slit. The gratings used were the 600 lines $/ \mathrm{mm}$ on the blue side, and a 316 lines $/ \mathrm{mm}$ one blazed at $7500 \AA$ on the red side, to produce a continuous $3700-10300 \AA$ spectrum, with typical spectral resolution of $4.9 \AA$ in the blue, and $3.9 \AA$ in the red. Details on the object are given in Section 3.2.1 and in the Appendix.

\subsection{Near-infrared Spectroscopy}

We observed WISEA 0615-1247 in the near-infrared with the upgraded SpeX spectrograph (Rayner et al. 2003) at the NASA Infrared $3 \mathrm{~m}$ Telescope Facility, on UT 2015 January 18. A low-resolution $(\lambda / \Delta \lambda=75-120)$ spectrum covering the range $0.69-2.54 \mu \mathrm{m}$ was obtained using the prism mode. Exposures were done in an ABBA nod pattern along the 15 arcsec slit; the total integration time was $956 \mathrm{~s}$. In order to apply telluric corrections, an A0 V star was observed, as close in airmass as possible to our target, although a 0.3 airmass difference was the best we could achieve. Data reduction was done with the SpeXtool package (Vacca et al. 2003; Cushing et al. 2004). Figure 3 shows the SpeX spectrum of WISEA 0615-1247. Wavelength regions of low atmospheric transmission (less than about $60 \%$ ) are indicated with gray bands. These regions correspond to water vapor absorptions at $0.92,1.13$, 1.35 , and $1.85 \mu \mathrm{m}$, as well as $\mathrm{CO}_{2}$ absorptions near 1.6, 2.0, and $2.06 \mu \mathrm{m}$ (Rayner et al. 2009). The spectrum exhibits two peaks at $\sim 1.08$ and $1.27 \mu \mathrm{m}$, which are near, but not at, the positions of $\mathrm{H}$ Paschen-series $\gamma$ and $\beta$ lines, respectively. The feature at $1.08 \mu \mathrm{m}$ is likely due to telluric $\mathrm{H}_{2} \mathrm{O}$, and in our experience is sometimes easier to see at the low spectral resolution of these data. The feature at $1.27 \mu \mathrm{m}$ is due to poor correction of a telluric $\mathrm{O}_{2}$ band.

\subsection{Previous Imaging}

We searched the literature for optical imaging data of WISEA 0615-1247. The purpose was to see if a background contaminant source may have been present in the beam during our optical spectroscopy of the object. The images examined were the $B$-band and $R$-band images in DSS2, and the $I$-band image in the Deep Near Infrared Survey of the Southern Sky 

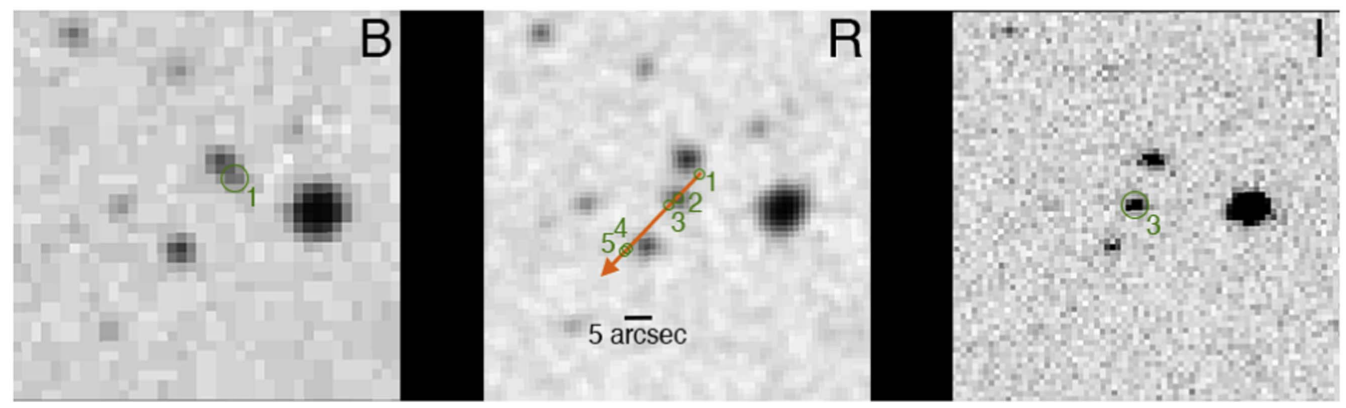

Figure 4. Image cut-outs $\left(66 \times 66 \operatorname{arcsec}^{2}\right)$ showing the positions of WISEA $0615-1247$ at various epochs. The left panel is the DSS2 B-band image, at epoch 1983 ; the middle panel is the DSS2 $R$-band image, at epoch 1993; and the right panel is the DENIS $I$-band image, at epoch 1999. The positions of WISEA 0615-1247 are indicated by the overlaid green circles and numbered labels 1 through 5, which correspond, respectively, to the $B$-band, $R$-band, $I$-band, AllWISE, and our optical spectroscopy epochs. The orange arrow overlaid on the $R$-band image shows the motion of WISEA $0615-1247$ throughout these epochs. The source just below the motion vector and to the right of the AllWISE position (number 4) is a neighboring DENIS Catalog non-moving source.

(DENIS, 3rd Data Release ${ }^{11}$ ). Figure 4 shows these three images.

Overlaid in Figure 4 are the positions of WISEA 0615 -1247 , shown as green circles and numbered labels, at respectively the $B$-band (1983), $R$-band (1993), $I$-band (1999), AllWISE (2010), and our optical spectroscopy (2014) epochs. The latter were computed from the AllWISE position of the object, and our estimate of motion from the displacement between 2MASS and AllWISE (Section 2.1). The orange arrow overlaid on the $R$-band image shows the motion of WISEA 0615-1247. The source just below the motion vector and to the right of the AllWISE position (number 4) is a neighboring DENIS Catalog non-moving source. This DENIS source was 4 or 5 arcsec from WISEA 0615-1247 during our spectroscopic observations, and hence did not contaminate our spectrum since our slit width was 1.0 arcsec. No other sources with strong $B$ - or $R$-band flux are seen in the field of our object at earlier epochs, hence we conclude that there is no contamination of our spectrum by a background source.

\subsection{Search for Other Archival Observations}

There were no X-ray measurements of this object in the ROSAT All Sky Survey ${ }^{12}$, or UV measurements in GALEX ${ }^{13}$, or radio measurements in the VLA Faint Images of the Radio Sky at Twenty-cm (FIRST) Survey ${ }^{14}$.

\section{POSSIBLE EXPLANATIONS}

Our repeated optical spectra of WISEA 0615-1247 measured throughout 2014 (Figure 2) do not show statistically significant evidence of variability. The spectral slopes are very comparable among the various observations (although the LRIS 2014 October 20 spectrum was modified, as described in Section 2.2).

\subsection{Single Object Hypothesis}

In order to compare our spectra of WISEA 0615-1247 with those of M dwarfs, we first combined our optical and near-

\footnotetext{
11 The DENIS Project website, including access to imaging data, is at http:// cdsweb.u-strasbg.fr/denis.html.

12 See the ROSAT All Sky Survey page at http://www.xray.mpe.mpg.de/cgi$\mathrm{bin} / \mathrm{rosat} /$ rosat-survey.

13 Information about GALEX is in http://www.galex.caltech.edu/ and http:// galexgi.gsfc.nasa.gov/.

14 The FIRST Survey is described in http://sundog.stsci.edu/.
}

infrared data into a single spectrum. We used our UT 2014 October 24 DBSP spectrum, which has the best signal-to-noise, and normalized our SpeX spectrum to it at $7916 \AA$. We then trimmed the two spectra into contiguous but non-overlapping ranges (4033-8764 A for DBSP, and 8783-24950 A for SpeX), taking into account that the optical red portion of the SpeX spectrum had better signal-to-noise than the DBSP one. The combined DBSP and SpeX spectrum is shown replicated in black in Figure 5, and is compared with spectra of M5 through M9 dwarfs from the SpeX Prism Spectral Libraries ${ }^{15}$ (Burgasser 2014), shown in red in Figure 5.

At wavelengths $>7000 \AA$, Figures 2 and 5 show that an M7 dwarf is the best overall fit to our spectrum, among the mid- to late-M dwarfs considered, but with a deficit of flux at $9000 \AA$ to $1.3 \mu \mathrm{m}$. Our spectrum exhibits numerous atomic and molecular features (Ca I, Ca II, TiO, and VO) indicating an M dwarf of solar metallicity (Figure 2). The spectrum also exhibits moderate $\mathrm{H} \alpha$ and $\mathrm{H} \beta$ emission lines. The former $(\mathrm{H} \alpha)$, absorption features of VO $(7300 \AA)$ and TiO $(8850 \AA)$, and an absorption line of Ca I (8500 $\AA$ ) suggest a late-M dwarf classification (Kirkpatrick 2009). The near-infrared spectrum (Figure 3) shows pronounced absorption bands of $\mathrm{H}_{2} \mathrm{O}$, wider than telluric ones, at $1.36-1.57$ and $1.72-2.1 \mu \mathrm{m}$, also indicative of a late-M dwarf classification (Kirkpatrick 2009).

In the blue optical, 4000-7200 $\AA$, the spectral slopes of the M7 dwarf shown in Figure 2, or M dwarfs shown in Figure 5, do not fit the spectral slope of our object. There is a prominent $\mathrm{Na} D$ absorption line at $5900 \AA$ in our object, that is not comparable in its intensity and width to that in $\mathrm{M}$ or $\mathrm{L}$ dwarfs. An early L-type dwarf spectrum would exhibit $\mathrm{Na} D$ absorption, although broader and of very different morphology than in our object. It would also exhibit features not seen in the spectrum of our object, most notably a prominent absorption feature from a K I doublet at $7750 \AA$, or absorption features from $\mathrm{MgH}(5200 \AA)$ and $\mathrm{CaOH}(5500 \AA)$, among others (Kirkpatrick 2009); see Figure 6.

The red optical spectra of WISEA 0615-1247 and an M7 dwarf are very different from those of late (M)-type subdwarfs (sdM), extreme subdwarfs (esdM), and ultra subdwarfs (usdM). The 7800-8100 $\AA$ spectral slope of an sdM8-9 is more smoothly monotonic in comparison with an M7 dwarf (Kirkpatrick et al. 2016; see their Figures 62 and 39). The corresponding slope of an esdM8-8.5 or a usdM8.5-9 is much

\footnotetext{
${ }^{15}$ The SpeX Prism Spectral Libraries are at http://browndwarfs.org/ spexprism/.
} 


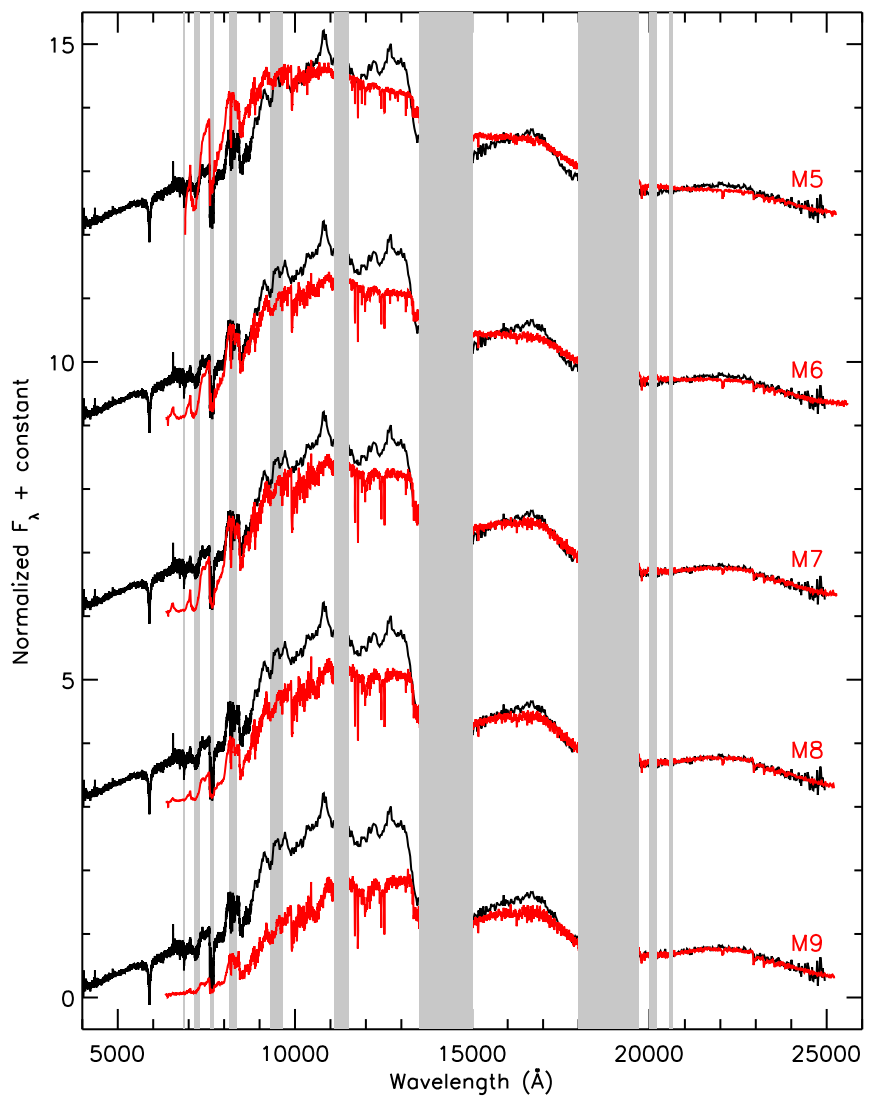

Figure 5. Black lines are the combination of our DBSP spectrum of WISEA 0615-1247 measured on UT 2014 October 24, trimmed to the range of 4033 through $8764 \AA$, and our SpeX spectrum trimmed to the range of $8783 \AA$ through $2.495 \mu \mathrm{m}$. The SpeX spectrum was normalized to the DBSP one at $7916 \AA$, before trimming. The combined spectrum, in normalized flux density units, was replicated vertically by adding offsets, for comparison with spectra of known mid- through late-M-type dwarfs (shown as red lines) from the SpeX Prism Spectral Libraries; see the text for reference. These comparison spectra were normalized to our object spectrum at $2.1 \mu \mathrm{m}$.

bluer than for an M7 dwarf (Kirkpatrick et al. 2016; see their Figures 71 and 74). Those same figures show that the 8500-9250 $\AA$ spectral region is very red in an M7 dwarf, while it is modestly red or flat in an sdM8-9, and turns over blue with increasing wavelength in an esdM8-8.5, or usdM8.5-9 (Kirkpatrick et al. 2016). The spectra of sdM8-9, esdM8-8.5, or usdM8.5-9 exhibit a very dramatic pseudocontinuum bump or $\mathrm{CaH}$ absorption trough at $6800 \AA$, in contrast to a barely noticeable feature in an M7 dwarf (Kirkpatrick et al. 2016), owing to TiO bands that swamp the $\mathrm{CaH}$ trough in the latter. Therefore, a late-type sdM, esdM, or usdM cannot reproduce the red optical spectrum of WISEA 0615-1247 while a solar metallicity M7 dwarf does.

\subsection{Binary Hypothesis}

In this section, we explore the possibility that an unresolved binary fits the data best. The binary would be comprised of an object responsible for the blue optical portion of the spectrum, while a late-M dwarf is largely responsible for the red optical and near-infrared portions.

The spectral energy distribution (SED) of a possible binary companion to a late-M dwarf can be assessed by subtracting a spectrum of the latter, from that of WISEA 0615-1247. Our

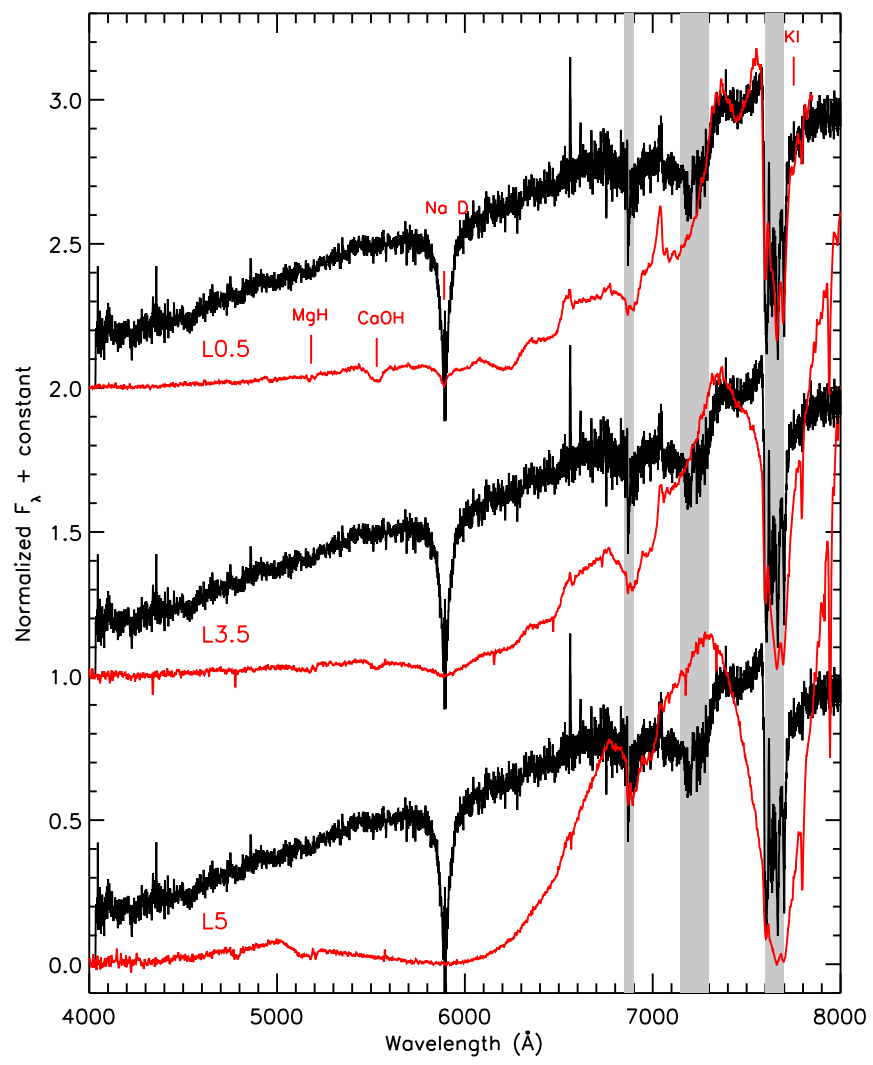

Figure 6. Black lines are our DBSP spectrum of WISEA 0615-1247 measured on UT 2014 October 24. The spectrum is plotted in normalized flux density units, and was replicated vertically by adding offsets, for comparison with spectra of known early- to mid-L type dwarfs (shown as red lines). These spectra are of 2MASS J07464256+2000321 (L0.5), 2MASS J00361617 +1821104 (L3.5), and 2MASS J15074769-1627386 (L5), from Kirkpatrick et al. (2000) and Reid et al. (2000). The spectra are plotted only up to $8000 \AA$ because of large discrepancies with our object at longer wavelengths. Regions of low atmospheric transmission (Hamuy et al. 1994) are indicated by gray bands. Feature identifications are shown in red for the L0.5 dwarf spectrum.

goals for the difference spectrum were for it to be positive in the optical, except in telluric regions, and to eliminate the $\mathrm{CaH}$ and $\mathrm{TiO}$ spectral features at 6750-7050 $\AA$, under the assumption that they are produced only by the late-M dwarf. The one parameter that we varied was the normalization factor of the late-M dwarf spectrum at a fixed wavelength, which then determined the corresponding normalization factor of the binary companion spectrum. We chose $7400 \AA$ as the normalization wavelength, because it is not in a telluric region, and both the spectrum of our object and a late-M dwarf are relatively featureless thereat. After experimenting with various normalization factors, we chose to normalize the late-M dwarf spectrum to $55 \%$ of the flux of our object at the above wavelength. Figure 7 shows the subtraction of the M7 dwarf spectrum in Figure 2, from our object's DBSP optical spectrum of 2014 October 24. Figure 7 shows that the $\mathrm{CaH}$ and $\mathrm{TiO}$ features were not eliminated, but our choice of normalization minimized their intensity. For normalization factors below $55 \%$, the above $\mathrm{CaH}$ and $\mathrm{TiO}$ features were more prominent, and for normalization factors above $55 \%$, negative fluxes resulted in various wavelength regions apart from telluric ones. Figure 7 shows that the spectrum of a possible companion to a late-M dwarf linearly rises from 4000 to $6700 \AA$, except that it exhibits a broad absorption feature of $\mathrm{Na}$ I D at $5900 \AA$. The 


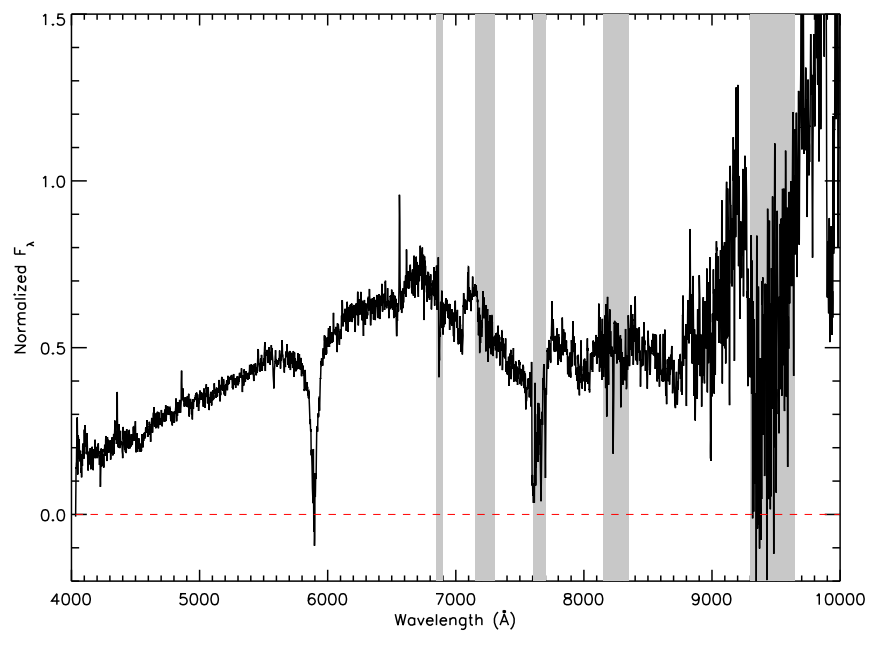

Figure 7. Difference between our 2014 October 24 spectrum of WISEA 0615 -1247 , and the spectrum of the M7 V star WISEA 1240+2047 (both shown in Figure 2). The M7 V star was normalized to $55 \%$ of our object's flux density at $7400 \AA$, before subtraction, as explained in Section 3.2. The resulting difference has been smoothed with a 3 pixel boxcar. The red dashed line indicates a zero difference. Regions of low atmospheric transmission (Hamuy et al. 1994) are indicated by gray bands.

spectrum then precipitously drops from $\sim 6700$ to $7600 \AA$. It is very roughly flat from $\sim 7700$ to $8800 \AA$, and finally rises in the remaining reddest part of it.

The difference spectrum in Figure 7, and particularly the excess in the optical blue, is plausibly produced by an object hotter than an $\mathrm{M}$ dwarf, and of relatively small radius, for its flux to be of the same order of magnitude as the M dwarf. Therefore, we considered a cool white dwarf as a possibility. Cool white dwarfs are those with temperatures $\lesssim 4500 \mathrm{~K}$. They are sometimes uncovered in proper motion surveys (Scholz et al. 2009, and references therein). Their infrared flux densities are depressed due to collision-induced absorption (CIA) by $\mathrm{H}_{2}$ (Saumon et al. 1994; Hansen 1998; Abel et al. 2011). We considered CIA mainly from $\mathrm{H}_{2}-\mathrm{H}_{2}$ pairs, such as in the models by Abel et al. (2011). However, depending on the relative abundance of $\mathrm{He}$, CIA from $\mathrm{H}_{2}-\mathrm{He}$ pairs may also be significant, as in models by Borysow et al. (1997). The effect of CIA is most evident at wavelengths $>1 \mu \mathrm{m}$. However, Oppenheimer et al. (2001) noted that the optical colors of cool white dwarfs are also affected by CIA. The opacity of CIA starts at approximately $0.6 \mu \mathrm{m}$ and increases toward the nearinfrared (Borysow et al. 1997). Models of cool white dwarf spectra with CIA, by D. Saumon and D. Koester (presented by Kilic et al. 2006), show that their SEDs are bluer and depressed relative to those of blackbodies. These models show that the onset of CIA in the optical is gradual, and its exact starting wavelength is difficult to quantify.

A cool white dwarf, as a companion to an M7 dwarf, might qualitatively explain the colors $J-K_{s}, J-W 2$ of WISEA 0615-1247 (Figure 1). CIA models of cool white dwarfs have bumps and wiggles in the infrared, rather than a smooth flux descent with increasing wavelength (D. Saumon and D. Koester, presented by Kilic et al. 2006; Abel et al. 2011). The $J-K_{s}$ color is similar to that in a Rayleigh-Jeans spectrum, while longer wavelength colors are redder. The $J-K_{s}$ color of WISEA 0615-1247 (Section 1) is $\sim 0.06 \mathrm{mag}$ bluer than $J-K_{s} \sim 1.0$ for a typical M7 dwarf (Figure 14 of Kirkpatrick et al. 2008). The $J-W 2$ color of WISEA 0615
-1247 (Section 1 ) is $\sim 0.18$ mag redder than $J-W 2 \sim 1.5$ for a typical M7 dwarf (Figure 7 of Kirkpatrick et al. 2011). We discuss these colors further at the end of Section 3.2.2.

\subsubsection{Comparison Cool White Dwarfs}

We searched the literature for spectra of cool white dwarfs with which to fit the difference spectrum in Figure 7. The cool white dwarfs we selected are SDSS J133001.13+643523.8, WD 2356-209, LHS 3250, WISEA 1702+7158B, and WD J0205-053. The first two show Na D absorption features, while the last three do not, although they were considered as potential fits to the continuum of the difference spectrum in the blue optical. Table 2 lists observational and physical characteristics of these five cool white dwarfs, and we also describe their spectroscopic and other characteristics next.

1. SDSS J133001.13+643523.8 (Harris et al. 2003) is a Heatmosphere white dwarf whose optical spectrum exhibits metallic lines. Its Sloan Digital Sky Survey (SDSS; York et al. 2000) spectrum has prominent and very broad Na I D absorption near $5892 \AA$. The SDSS spectrum, of very low signal-to-noise, tentatively exhibits $\mathrm{Ca}$ I absorption features at 4226,4335 , and $4455 \AA$, and Ca II infrared triplet absorption features at 8498, 8542, and $8602 \AA$ (Harris et al. 2003). Unlike the spectra of other DZ white dwarfs, that of SDSS J133001.13+643523.8 does not exhibit the Mg I triplet lines near $5175 \AA$, or the Ca II H \& K lines (3968 and $3934 \AA$ A).

2. WD 2356-209 was discovered as a candidate Galactic halo cool white dwarf by Oppenheimer et al. (2001). It is a DZ-type white dwarf, and its optical spectrum shows a broad and prominent absorption feature from $\mathrm{Na}$ I D and possible absorption features from the $\mathrm{Mg}$ I triplet near $5175 \AA$ (Oppenheimer et al. 2001).

3. LHS 3250 (Luyten 1976) was discovered to be the first cool white dwarf with CIA by Harris et al. (1999). It is a DC-type white dwarf, meaning it has no discernible spectral features in the optical.

4. LSPM J1702+7158N (Lépine \& Shara 2005) ${ }^{16}$ is the secondary-component of a common-proper motion binary (10". 8 separation); the primary component is LP 43-310 (Salim \& Gould 2003; Lépine \& Shara 2005). Kirkpatrick et al. (2016) rediscovered the common-proper motion system in an analysis of AllWISE high motion sources. Kirkpatrick et al. (2016) obtained a DBSP spectrum of LSPM J1702+7158N and they classified the object as a cool white dwarf.

5. WD J0205-053 was also discovered as a candidate Galactic halo cool white dwarf by Oppenheimer et al. (2001), and was followed up through optical photometry and spectroscopy by Salim et al. (2004). No spectral lines are seen in the optical, hence its classification as DC-type. Salim et al. (2004) concluded it had a He atmosphere. However, the SED of a pure-He atmosphere white dwarf would be expected to be nearly identical to that of a blackbody (Kowalski \& Saumon 2006). Bergeron et al. (2005) obtained optical and near-infrared ground-based photometry, and Kilic et al. (2009) obtained Spitzer IRAC photometry of this and other similar objects. Kilic et al. (2009) fitted atmospheric models to these data, and

\footnotetext{
16 The LSPM-North Catalog is accessible at https://heasarc.gsfc.nasa.gov/ W3Browse/all/lspmnorth.html.
} 
Table 2

Characteristics of Comparison Cool White Dwarfs

\begin{tabular}{|c|c|c|c|c|c|c|}
\hline $\begin{array}{l}\text { Name } \\
\text { (1) }\end{array}$ & $\begin{array}{l}\text { Type } \\
\text { (2) }\end{array}$ & $\begin{array}{c}\text { Temperature } \\
\text { (K) } \\
\text { (3) }\end{array}$ & $\begin{array}{l}\text { Distance } \\
\text { (pc) } \\
(4)\end{array}$ & $\begin{array}{c}\mu_{\alpha} \\
\left(\operatorname{mas~yr}^{-1}\right) \\
(5)\end{array}$ & $\begin{array}{c}\mu_{\delta} \\
\left(\operatorname{mas~yr}^{-1}\right) \\
(6)\end{array}$ & Ref. $^{\text {a }}$ \\
\hline SDSS J133001.13+643523.8 & $\mathrm{DZ}$ & $\cdots$ & $\cdots$ & -193 & 0 & 1 \\
\hline LHS 3250 & DC & $3000-4000$ & 30 & $-548.1 \pm 5.3$ & $157.3 \pm 5.3$ & 3 \\
\hline LSPM J1702+7158N & $\ldots$ & $\ldots$ & $\ldots$ & $-286.5 \pm 13.1^{\mathrm{c}}$ & $119.3 \pm 7.3^{\mathrm{c}}$ & 4 \\
\hline WD J0205-053 & $\mathrm{DC}^{\mathrm{d}}$ & $4200^{\mathrm{d}}$ & $29 \pm 8 \mathrm{pc}$ & \multicolumn{2}{|c|}{$1051 \pm 25^{\mathrm{e}}$} & 2 \\
\hline
\end{tabular}

Notes.

${ }^{a}$ References to discoveries: (1) Harris et al. (2003). (2) Oppenheimer et al. (2001). (3) Harris et al. (1999) rediscovered by Harris et al. (2001). (4) Lépine \& Shara (2005).

${ }^{\mathrm{b}}$ Distance and temperature estimates by Salim et al. (2004); Oppenheimer et al. (2001) estimate a distance of 85 pc; Bergeron et al. (2005) esimate a distance of 77 pc and a temperature of $4790 \mathrm{~K}$.

${ }^{c}$ 2MASS-to-AllWISE proper motion estimates by Kirkpatrick et al. (2016). The motion from the AllWISE Catalog is R.A. motion $=-303 \pm 46$ mas yr ${ }^{-1}$ and decl.

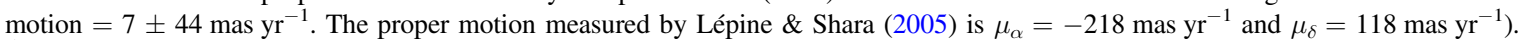

${ }^{\mathrm{d}}$ Spectral type and temperature estimates by Salim et al. (2004), very comparable to estimates by Bergeron et al. (2005) and Kilic et al. (2009).

e The R.A. and decl. components of motion are not available from Oppenheimer et al. (2001).

WD J0205-053 itself was modeled with a pure-H atmosphere. Kilic et al. (2009) found that, in their sample of $44 \mathrm{cool}$ white dwarfs, only one had a pure-He atmosphere.

We also selected for comparison the white dwarf WD 2213 +317 , whose coordinates in SIMBAD associate it with the AllWISE high motion object WISEA 221515.51+315731.9 (Kirkpatrick et al. 2016). Kirkpatrick et al. (2016) found that the colors $J-K_{s}=0.889 \pm 0.025$ mag and $J-W 2=1.081 \pm 0.028 \mathrm{mag}$ place the AllWISE object among normal $\mathrm{M}$ dwarfs in color space (Figure 1). Kirkpatrick et al. (2016) pointed to other binary systems known from the literature, consisting of $\mathrm{M}$ and white dwarfs, which also fall at the location of normal M dwarfs, and suggested that WD 2213 +317 was worthy of follow-up. Unfortunately, the SIMBAD association of the white dwarf and the AllWISE object is erroneous; the latter is an early-M-type dwarf instead, as explained in the Appendix.

\subsubsection{Binaries of Late-M and Cool White Dwarfs}

Figures 8 and 9 show the comparison of our spectrum of WISEA 0615-1247 with spectral binary templates constructed from the above cool white dwarfs and an M7 dwarf. Spectra of the two cool white dwarfs with $\mathrm{Na}$ I D absorption listed in Section 3.2.1, SDSS J133001.13+643523.8 and WD 2356 -209 , are shown in Figure 8, and spectra of the other three cool white dwarfs are shown in Figure 9. The spectra of the binary components were each normalized at $7400 \mu \mathrm{m}$. Their relative contributions were empirically determined by attempting to fit, after their addition, the blue optical spectral slope, the red optical detailed SED and spectral features, and by avoiding flux densities in excess of the observed spectrum of WISEA 0615 -1247. After experimentation, we obtained fits using the fractions listed in the captions of Figures 8 and 9.

Figure 8 shows that the $\mathrm{Na}$ I D absorption in the two cool white dwarfs is substantially broader than in WISEA 0615 -1247 . It must be that pressure broadening is more

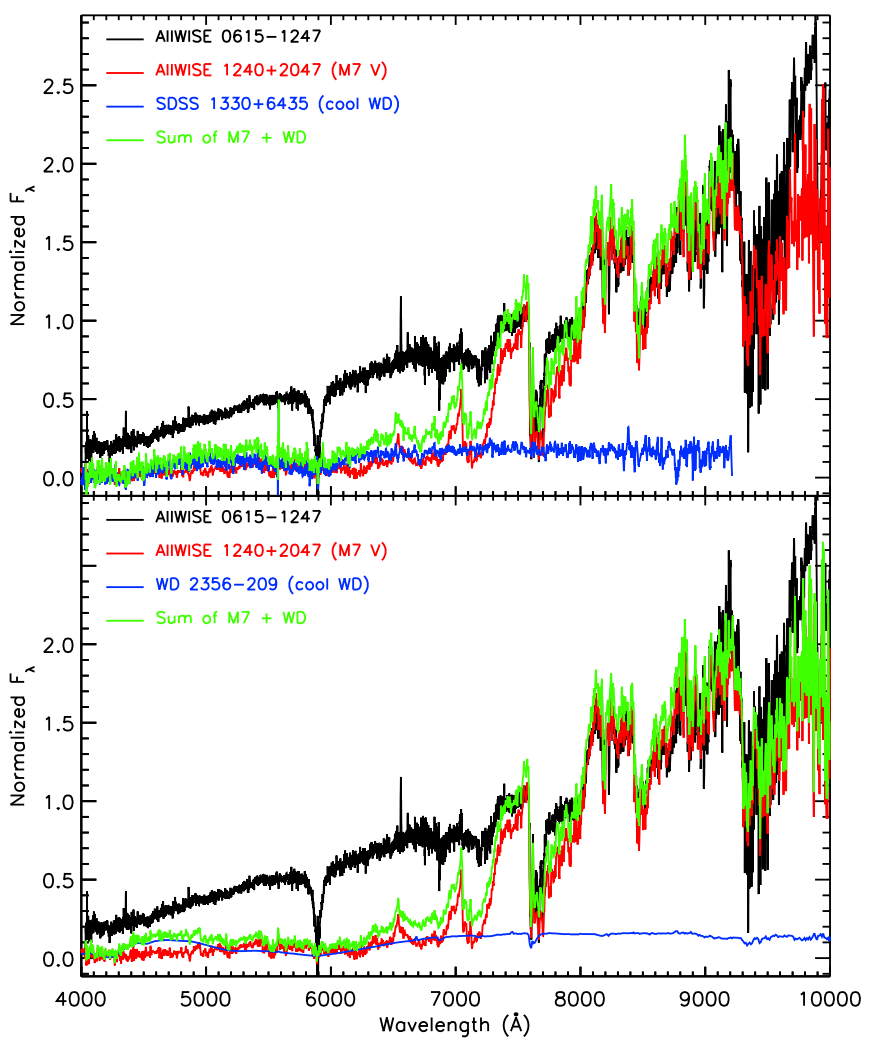

Figure 8. Comparison of our optical (DBSP) spectrum of WISEA 0615-1247 from UT 2014 October 24 (shown in black), with the addition of an M7 dwarf spectrum (shown in red, as in Figure 2), and, respectively, two cool white dwarf spectra (shown in blue). The upper panel includes the SDSS spectrum of SDSS J133001.13+643523.8 (Harris et al. 2003), smoothed with a 5 pixel boxcar. The lower panel includes the LRIS spectrum (unpublished data courtesy of S. Salim; see Salim et al. 2004 for details) of WD 2356-209 (Oppenheimer et al. 2001). Both cool white dwarf spectra were separately normalized to $15 \%$ of the observed $7400 \AA$ flux density of the WISEA 0615 -1247 spectrum, and the M7 dwarf spectrum was normalized to the remainder $(85 \%)$ of this flux density. These empirical fractions were estimated as described in the text. The addition of each cool white dwarf and M7 dwarf spectra, linearly interpolated to a common wavelength grid, is shown in green. 


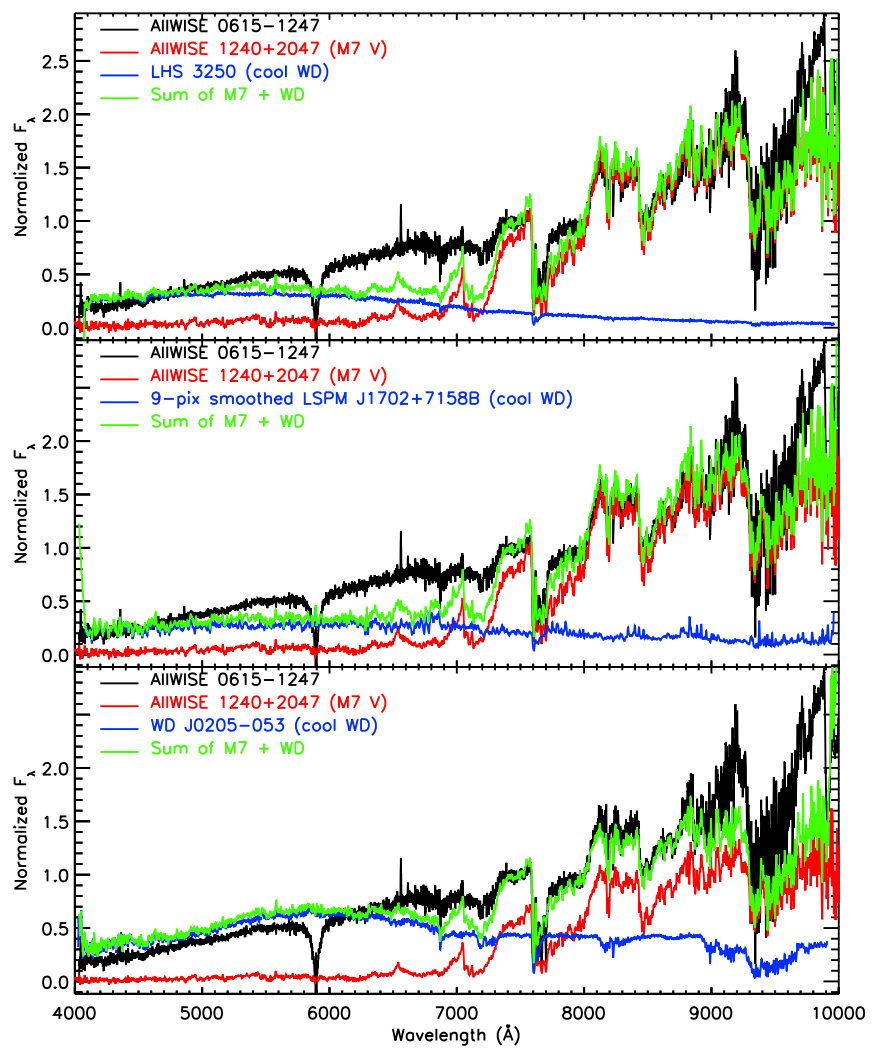

Figure 9. Comparison of our optical (DBSP) spectrum of WISEA 0615-1247 from UT 2014 October 24 (shown in black), with the addition of an M7 dwarf spectrum (shown in red, as in Figure 2), and, respectively, three cool white dwarf spectra (shown in blue). The upper panel includes our DBSP spectrum of LHS 3250 (Harris et al. 2001). The middle panel includes the Kirkpatrick et al. (2016) DBSP spectrum of LSPM J1702+7158N (Lépine \& Shara 2005). The lower panel includes our DBSP spectrum of WD J0205-053 (Oppenheimer et al. 2001). The three cool white dwarf spectra were separately normalized to $15 \%, 20 \%$, and $45 \%$, respectively, of the observed $7,400 \AA$ flux density of the WISEA 0615-1247 spectrum, and the M7 dwarf spectrum was normalized to the remainder $(85 \%, 80 \%$, and $55 \%$, respectively) of this flux density. These empirical fractions were estimated as described in the text. The addition of each cool white dwarf and M7 dwarf spectra, linearly interpolated to a common wavelength grid, is shown in green.

pronounced in these cool white dwarfs, than in our system. The spectral energy distributions (SEDs) of these two cool white dwarfs are not an adequate fit as binary companions in this case, because they produce too much flux in the red optical, and not enough in the blue. Conversely, the red spectrum is mostly fit by an M7 dwarf. CIA in these cool white dwarfs appears to be less than in our system, possibly due to higher effective temperatures. We note that the temperature estimate of WD 2356-209 by Bergeron et al. (2005; see footnote (b) in Table 2) is relatively high. However, the models by Bergeron et al. (2005) tend to yield relatively high temperature values $(\mathrm{H}$. Harris 2016, private communication).

In Figure 9, we show DBSP spectra for three cool white dwarfs; two of them (LHS 3250 and WD J0205-053) were originally measured in the SDSS, but our new spectra have better signal-to-noise; the spectrum of the other source (LSPM $\mathrm{J} 1702+7158 \mathrm{~N}$ ) was measured by Kirkpatrick et al. (2016). Figure 9 shows that the spectra of the three cool white dwarfs are featureless in the optical blue, thus not being adequate models for the Na I D absorption in our system. The spectra of these three cool white dwarfs, however, have diminishing flux with increasing wavelength in the optical red, likely from strong CIA. These spectra contribute significantly to the hybrid spectrum in the optical blue. The above fits, however, are only approximately successful in the interval 4000-4800 $\AA$ for LHS 3250 (upper panel in Figure 9) and 4000-4600 $\AA$ for LSPM $\mathrm{J} 1702+7158 \mathrm{~N}$ (middle panel in Figure 9). In particular, the slopes of the combined (binary) spectra (green lines in the upper and middle panels in Figure 9) are slightly bluer than in the spectrum of our system (black lines in this figure). The fit of the spectrum of WD J0205-053 (lower panel in Figure 9), when added to the same M7 dwarf, however, is quite promising. This fit reproduces our spectral slope in the interval 4000-5800 $\AA$, although with a small excess of flux. Other limitations of this fit (green line in the bottom panel of Figure 9) are a flux deficit at 6200-7300 $\AA$ or, equivalently, that the slope turnover due to CIA occurs at a redder wavelength in our system, namely $\sim 6800 \AA$, than in WD J0205-053 ( $\sim 6000 \AA)$; a deficit of flux in the optical red; and most notably lack of $\mathrm{Na}$ I D absorption. It is possible that the cool white dwarf in WISEA 0615-1247 has a higher abundance of $\mathrm{Na}$ than in WD J0205-053.

To check the consistency of our empirical relative flux normalizations of an M7 dwarf and a cool white dwarf, such as WD J0205-053, we first estimated synthetic broadband $V$ magnitudes from their spectra. We then obtained absolute $V$ magnitudes $M_{V}$ of these objects from the literature, and compared the resulting relative fluxes. The spectra of the M7 dwarf and WD J0205-053 in Figure 9, when convolved with the Johnson-Morgan $V$ filter transmission function (Mermilliod et al. 1997), ${ }^{17}$ indicate that the cool white dwarf is a factor of $\sim 14$ brighter than the M7 dwarf. ${ }^{18}$ On the other hand, an M7 dwarf has $M_{V} \sim 17.81$ (from an online tabular compilation ${ }^{19}$ referred to by Pecaut \& Mamajek 2013), while the cool white dwarf WD J0205-053 has $M_{V} \sim 16.59$ (given its distance and apparent $V$ magnitude). Therefore, at a common distance from us, the cool white dwarf is predicted to be a factor of $\sim 3.1$ brighter at $V$ band than an M7 dwarf. It is possible that the cool white dwarf in the WISEA 0615-1247 system is hotter than WD J0205-053, or has a larger radius, as discussed at the end of this section, for it to be so much brighter than the M7 dwarf.

An estimate of the photometric distance to WISEA 0615 -1247 can be obtained in view that the cool white dwarf is comparatively much fainter than an M7 dwarf at $J$ band. An M7 dwarf has an absolute magnitude of $M_{J} \sim 10.8$ (Hawley et al. 2002; online compilation (see footnote 19) referred to by Pecaut \& Mamajek 2013), and WD J0205-053 has $M_{J} \sim 14.4$ (from its distance and 2MASS $J$ ), resulting in a flux ratio of $\sim 27$. Neglecting the contribution from the cool white dwarf, the distance to our system is $\sim 58 \mathrm{pc}$, per the observed 2MASS $J$ magnitude (Table 1).

The above distance to WISEA 0615-1247 and its proper motion estimated from 2MASS and AllWISE (Section 2.1) imply a tangential velocity of $\sim 165 \mathrm{~km} \mathrm{~s}^{-1}$. We assessed the possibility that this velocity suggested a subdwarf in our system, even though our spectra are not consistent with a

\footnotetext{
17 Johnson-Morgan-Cousins UVBRI filter transmission functions are in the General Catalog of Photometric Data, at http://obswww.unige.ch/GCPD/.

18 The synthetic $V$ magnitude we obtained from the spectrum of WISEA 0615 -1247 is $20.4 \mathrm{mag}$, which differs by $0.8 \mathrm{mag}$ from the estimate by Lépine (2008), listed in Table 1. The latter was derived from photographic magnitudes, using color-color models for field dwarfs, unreliable for an unusual binary system such as WISEA 0615-1247, and is subject to systematic errors and other effects (Lépine 2008).

19 See http://www.pas.rochester.edu/ emamajek.
} 
subdwarf (Section 3.1). Traditionally, subdwarfs were considered to be in the galactic halo (Ryan \& Norris 1991; Gizis 1997; Lépine et al. 2003, 2007), and therefore dynamically "heated." The median tangential velocity in the galactic halo is $\sim 220 \mathrm{~km} \mathrm{~s}^{-1}$ (Reid \& Hawley 2005), while the typical velocity in the galactic disk is $\sim 37 \mathrm{~km} \mathrm{~s}^{-1}$ (Reid 1997). The escape velocity of the Galaxy near the Sun is $\sim 525 \mathrm{~km} \mathrm{~s}^{-1}$ (Carney \& Latham 1987). In a recent study of the kinematics of 3517 M-type objects of metallicity classes sdM, esdM, and usdM, Savcheva et al. (2014) found that the sdMs tend to be at the galactic thick or old disk, while the esdMs and usdMs are at the galactic halo. A histogram of the tangential velocities of these objects showed a peak at $\sim 50 \mathrm{~km} \mathrm{~s}^{-1}$, and values almost reaching $600 \mathrm{~km} \mathrm{~s}^{-1}$ (Savcheva et al. 2014). The tangential velocity of WISEA $0615-1247$ is a factor of approximately four times larger than in the galactic thin disk, and approximately three times larger than in the thick disk, though smaller than in the halo. The metallicity of WISEA 0615-1247 is inconsistent with membership in the latter, but it is probable that it belongs to the thick disk. However, it should be taken into account that there are high excursions in the velocities of individual objects around the above typical values. Therefore, any definite conclusion on the galactic population membership of WISEA 0615-1247 will require a detailed kinematic study, including radial velocity and precise parallax measurements.

We computed the $J-K_{s}$ and $J-W 2$ colors of the binary system of an M7 dwarf and the cool white dwarf WD J0205 -053 , to see if they would reproduce the colors of WISEA $0615-1247$ shown in Figure 1 . We used $J$ and $K$ photometry of WD J0205-053 by Bergeron et al. (2005), and Spitzer IRAC channel 2 photometry by Kilic et al. (2009), which we regarded as equivalent to WISE W2, and computed absolute magnitudes. We used absolute magnitudes $M_{J}$ and $M_{K}$ of an M7 dwarf from the online reference by Pecaut \& Mamajek (2013; see footnote 20). However, the fact that the cool white dwarf is intrinsically much fainter than the M7 dwarf results in minute changes of colors $J-K_{s}$ and $J-W 2$ for this binary, relative to an M7 dwarf (Section 3.2), of $\sim 0.03$ and 0.05 mag, respectively.

A more luminous cool white dwarf would either have a higher effective temperature, or a larger radius, than WD J0205 -053 . However, the effects of these two possibilities on CIA have to be taken into account. Borysow et al. (1997) showed that CIA decreases with increasing effective temperature, and increases with surface gravity, and that CIA is pronounced for low metallicity $\left(Z<0.1 Z_{\odot}\right)$ and effective temperature up to $4000 \mathrm{~K}$. Bergeron et al. (2005) and Tremblay \& Bergeron (2008) computed pure-H atmospheric models of cool white dwarfs. In these models, the color $V-H$ becomes redder by $\sim 0.10-0.17$ mag when the effective temperature increases from $4200 \mathrm{~K}$ (as in models for WD J0205-053) to $4500 \mathrm{~K}$ (the temperature at which the above reddening effect, due to reduced CIA, is reversed). In our attempts to fit the SED of WISEA 0615-1247 with binaries, we assumed that most of the near-infrared flux is emitted by an M7 dwarf, hence constraining $V-H$ color variations to corresponding $V$ variations in the cool white dwarf. It follows that an increase in effective temperature has only a modest effect, and of opposite sense, in trying to solve the discrepancy in the predicted and observed flux ratios of the M7 and the cool white dwarf. In the models by Tremblay \& Bergeron (2008), the color $V-J$ becomes redder by $\sim 0.05 \mathrm{mag}$ when surface gravity $\log (g)$ decreases from 8.5 to 7.5 , at an effective temperature of $4500 \mathrm{~K}$. The reddening effect is $\sim 0.1 \mathrm{mag}$ at an effective temperature of $4200 \mathrm{~K}$. It follows that an increase in radius of a cool white dwarf leads to only a modest reduction in CIA, if $\log (g)$ variations are within the above range. The assumed surface gravity of cool white dwarf models of WD J0205-053 by Bergeron et al. (2005) and Kilic et al. (2009) is $\log (g)=8.0$. Surface gravity should remain close to this value, as a result of an increase in the radius of a cool white dwarf, so that CIA is still significant.

A cool white dwarf of large radius but of surface gravity comparable to that of WD J0205-053 may be hypothesized as a possibly improved fit, as a binary component, to WISEA 0615-1247. Althaus et al. (2009) ${ }^{20}$ modeled He-core, H-atmosphere white dwarfs, whose progenitors had supersolar metallicities. In these models, a $0.22 M_{\odot}$ object with $\log \left(Z / Z_{\odot}\right)=0.03$, and effective temperature of $4100-4200$ $\mathrm{K}$, has a surface gravity of $\log (g)=7.12, M_{V} \sim 15 \mathrm{mag}, B-V$ $\sim 1.04 \mathrm{mag}$, and $J-K \sim 0.2 \mathrm{mag}$. For comparison, the colors of WD J0205-053 from Bergeron et al. (2005) are $B-V=1.32 \mathrm{mag}$ and $J-K=0.1 \mathrm{mag}$, not too diifferent from those of the above Althaus et al. (2009) model white dwarf. A binary of this white dwarf and an M7 dwarf results in a shift of $J-K$ toward the blue of $\sim 0.07 \mathrm{mag}$, relative to an M7 dwarf. This shift is larger than for WD J0205-053, and comparable to that seen in WISEA 0615-1247 ( $~ 0.06 \mathrm{mag}$, Section 3.2).

\subsection{Other Possible Hypotheses}

Other scenarios we explored are as follows.

1. A triple system composed of two late-M dwarfs and a cool white dwarf. It is possible that a third component, in addition to the binary system we discussed in Section 3.2.2, can improve the spectral fit to our data, such as in covering the flux deficit we currently see in the interval 6200-7300. . If this third component is an sdM, esdM, or usdM, it would have to have been captured, because its low metallicity is disparate from the presence of $\mathrm{TiO}$ lines in our spectra. It is possible that an sdM, esdM, or usdM can improve the spectral fit in the nearinfrared. Figure 5 shows that an M7 dwarf spectrum yields flux deficits at 9700-11000 $\AA$ and 11500-13000 $\AA$, as well as a bluer slope at 15000-16500 $\AA$, and/or a deficit at $16800 \AA$, relative to WISEA 0615-1247. The relative flux contribution of an sdM, esdM, or usdM in a multiple system, that would alleviate these shortcomings, is difficult to assess. However, as described at the end of Section 3.1, an sdM, esdM, or usdM is not a good fit to the optical red spectrum of WISEA 0615-1247, and such remains the case regardless of the flux ratios in a multiple system. The bulk of the flux contribution in the optical red would have to be from an M7 dwarf.

2. There could be unusual chromospheric activity in a single or multiple stellar system. We would have expected emission features, in contrast to the pronounced and broadened Na I D absorption that we see. The UT 2014 October 24 spectrum exhibits modest $\mathrm{H}_{\alpha}$ and $\mathrm{H}_{\beta}$ lines, that might indicate some level of this kind of activity. However, white-light flare events in active $\mathrm{M}$ dwarfs, which occur due to strong magnetic fields, produce much

\footnotetext{
${ }^{20}$ See the online tabulation of models of white dwarfs by Althaus et al. (2009) at: http://evolgroup.fcaglp.unlp.edu.ar/TRACKS/tracks_heliumcore.html.
} 
stronger $\mathrm{H}$ Balmer series lines than seen in our object (Kowalski et al. 2010 and references therein), and there is no Na I D absorption.

\section{CONCLUSIONS}

We propose that WISEA 0615-1247 is an unresolved binary consisting of an M7 dwarf and a cool white dwarf, the latter having an SED unlike any other cool white dwarf currently known. We speculate that the cool white dwarf produces the observed $\mathrm{Na} \mathrm{I} \mathrm{D}$ absorption feature, if $\mathrm{Na}$ abundance is high, and if pressure broadening is less than in other known cool white dwarfs. Additional studies of this fascinating system are warranted, including an accurate trigonometric parallax measurement.

We thank our referee, Derek Homeier, for suggestions and corrections that greatly improved the manuscript. We also thank Hugh Harris and Samir Salim for very useful discussions that greatly benefited this paper, and Samir Salim for allowing us to use unpublished data. This publication makes use of data products from WISE, which is a joint project of the University of California, Los Angeles, and the Jet Propulsion Laboratory (JPL)/California Institute of Technology (Caltech), funded by the National Aeronautics and Space Administration (NASA). This research has made use of the NASA/Infrared Processing and Analysis Center (IPAC) Infrared Science Archive, which is operated by JPL/Caltech, under contract with NASA. This publication also makes use of data products from the Two Micron All Sky Survey, which is a joint project of the University of Massachusetts and IPAC/Caltech, funded by NASA and the National Science Foundation (NSF). The DENIS project has been partly funded by the SCIENCE and the HCM plans of the European Commission under grants CT920791 and CT940627. It is supported by INSU, MEN, and CNRS in France, by the State of Baden-Württemberg in Germany, by DGICYT in Spain, by CNR in Italy, by FFwFBWF in Austria, by FAPESP in Brazil, by OTKA grants F-4239 and F-013990 in Hungary, and by the ESO C\&EE grant A-04-046. Funding for the SDSS and SDSS-II has been provided by the Alfred P. Sloan Foundation, the Participating Institutions, NSF, the U.S. Department of Energy, NASA, the Japanese Monbukagakusho, the Max Planck Society, and the Higher Education Funding Council for England. The SDSS Web Site is http://www.sdss.org/. The SDSS is managed by the Astrophysical Research Consortium for the Participating Institutions. The Participating Institutions are the American Museum of Natural History, Astrophysical Institute Potsdam, University of Basel, University of Cambridge, Case Western Reserve University, University of Chicago, Drexel University, Fermilab, the Institute for Advanced Study, the Japan Participation Group, Johns Hopkins University, the Joint Institute for Nuclear Astrophysics, the Kavli Institute for Particle Astrophysics and Cosmology, the Korean Scientist Group, the Chinese Academy of Sciences (LAMOST), Los Alamos National Laboratory, the Max-Planck-Institute for Astronomy (MPIA), the Max-Planck-Institute for Astrophysics (MPA), New Mexico State University, Ohio State University, University of Pittsburgh, University of Portsmouth, Princeton University, the United States Naval Observatory, and the University of Washington. The Digitized Sky Surveys were produced at the Space Telescope Science Institute under U.S.

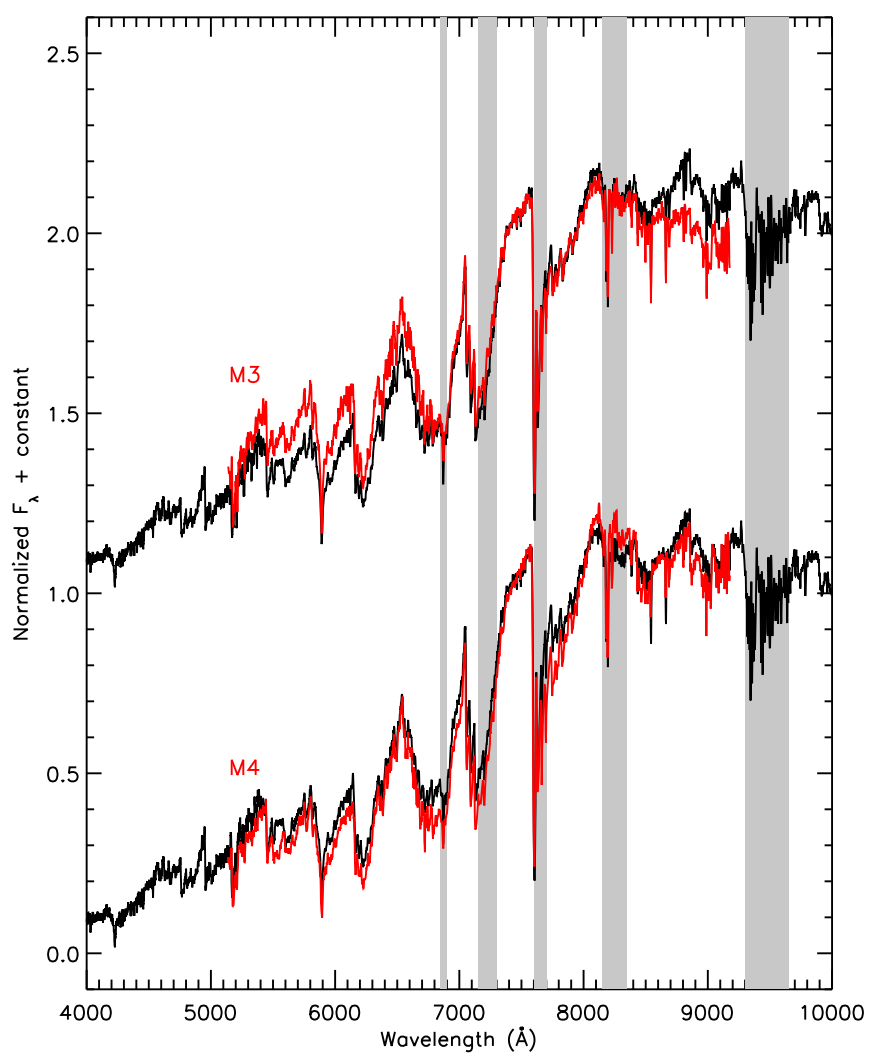

Figure 10. Black lines are our DBSP spectrum of LP 343-35, rediscovered as the high motion source WISEA 221515.51+315731.9 (Kirkpatrick et al. 2016).

The spectrum is plotted in normalized flux density units, and was replicated vertically by adding offsets, for comparison with DBSP spectra of known M3and M4-type dwarfs (shown as red lines). The object is of spectral type intermediate between M3 and M4. Regions of low atmospheric transmission (Hamuy et al. 1994) are indicated by gray bands.

Government grant NAG W-2166. The images of these surveys are based on photographic data obtained using the Oschin Schmidt Telescope on Palomar Mountain and the UK Schmidt Telescope. The plates were processed into the present compressed digital form with the permission of these institutions.

\section{APPENDIX \\ MISIDENTIFICATION OF THE WHITE DWARF WD $2213+317$ AS WISEA $221515.51+315731.9$}

The high motion object WISEA $221515.51+315731.9$ is a rediscovery by Kirkpatrick et al. (2016) of LP 343-35, listed in Luyten's White Dwarf Catalogs $(1970-1977)^{21}$ as a wide binary companion (more than 2 arcmin away) to LP 343-34. SIMBAD erroneously associates the coordinates of the AllWISE object to LP 343-34, which is the white dwarf WD $2213+317$. Based on the SIMBAD association, Kirkpatrick et al. (2016) suggested that the AllWISE object was an unresolved binary of a white and an M dwarf, upon which we measured its spectrum with DBSP (Figure 10) to investigate its nature. Figure 10 also shows comparison spectra of M3 and M4 dwarfs. This object is clearly an M3.5 dwarf.

We also investigated the putative binarity nature of LP 343-34 and 343-35. The AllWISE Source Catalog lists the motion of LP

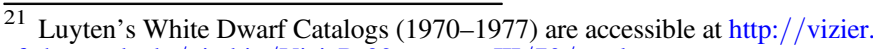
cfa.harvard.edu/viz-bin/VizieR-3?-source $=$ III/70/catalog.
} 
343-35 as R.A. motion $=-54 \pm 39$ mas yr$^{-1}$ and decl.

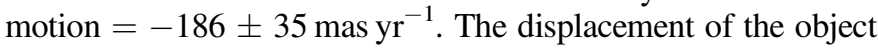
between its 2MASS and AllWISE positions yields $\mu_{\alpha}=3.3 \pm 6.4$ mas yr $^{-1}$ and $\mu_{\delta}=-111.8 \pm 6.3 \mathrm{mas} \mathrm{yr}^{-1}$ (Kirkpatrick et al. 2016), in rough agreement (within $3 \sigma$ ) with the AllWISE catalog motion. The object LP 343-34 = 2MASS $\mathrm{J} 22150696+3158402=\mathrm{LSPM} 2215+3158$ (Lépine \& Shara 2005) must be the white dwarf WD $2213+317$. Its listed proper motions in the LSPM-N Catalog are $\mu_{\alpha}=-47 \mathrm{mas} \mathrm{yr}^{-1}$, and $\mu_{\delta}=-149$ mas yr $^{-1}$ (Lépine \& Shara 2005). Luyten's White Dwarf Catalogs (1970-1977) list a total motion of $128 \mathrm{mas} \mathrm{yr}^{-1}$ at P.A. $=188^{\circ}$, consistent with the above. Our measurement of proper motion from the displacement between the 2MASS and AllWISE positions of LP 343-34 is $\mu_{\alpha}=-135.3 \pm$ $29.8 \mathrm{mas} \mathrm{yr}^{-1}$, and $\mu_{\delta}=-226.8 \pm 31.8 \mathrm{mas} \mathrm{yr}^{-1}$, in agreement, within $3 \sigma$, with the LSPM-N and Luyten's Catalogs. The latter described LP 343-34 and 343-35 as a common-proper motion binary. However, the motions of the two objects are distinct, as can be seen by comparing the 2MASS-to-AllWISEderived values. Therefore, the white dwarf WD 2213+317 and our discovered M3.5 dwarf are not physically associated.

\section{REFERENCES}

Abel, M., Frommhold, L., Li, X., \& Hunt, K. L. C. 2011, JPCA, 115, 6805 Althaus, L. G., Panei, J. A., Romero, A. D., et al. 2009, A\&A, 502, 207 Bergeron, P., Ruiz, M. T., Hamuy, M., et al. 2005, ApJ, 625, 838 Borysow, A., Jorgensen, U. G., \& Zheng, C. 1997, A\&A, 324, 185 Burgasser, A. J. 2014, in ASI Conf. Ser. 11, Int. Workshop on Stellar Spectral Libraries, ed. H. P. Singh, P. Prugniel, \& I. Vauglin (Bengaluru: ASI), 7

Carney, B., \& Latham, D. 1987, in IAU Symp. 117, Dark Matter in the Universe, ed. J. Kormendy \& G. Knapp (Dordrecht: Reidel), 39

Cushing, M. C., Vacca, W. D., \& Rayner, J. T. 2004, PASP, 116, 362

Gizis, J. 1997, AJ, 113, 806

Hamuy, M., Suntzeff, N. B., Heathcote, S. R., et al. 1994, PASP, 106, 566 Hansen, B. M. S. 1998, Natur, 394, 860

Harris, H. C., Dahn, C. C., Vrba, F. J., et al. 1999, ApJ, 524, 1000

Harris, H. C., Hansen, B. M. S., Liebert, J., et al. 2001, ApJL, 549, L109

Harris, H. C., Liebert, J., Kleinman, S. J., et al. 2003, AJ, 126, 1023

Hawley, S. L., Covey, K. R., Knapp, G. R., et al. 2002, AJ, 123, 3409
Kilic, M., Kowalski, P. M., Reach, W. T., \& von Hippel, T. 2009, ApJ, 696, 2094

Kilic, M., von Hippel, T., Mullally, F., et al. 2006, ApJ, 642, 1051

Kirkpatrick, J. D. 2009, in Stellar Spectral Classification, ed. R. O. Gray \& C. J. Corbally (Princeton: Princeton Univ. Press), 339

Kirkpatrick, J. D., Cruz, K. L., Barman, T. S., et al. 2008, ApJ, 689, 1295

Kirkpatrick, J. D., Cushing, M. C., Gelino, C. R., et al. 2011, ApJS, 197, 19

Kirkpatrick, J. D., Kellogg, K., Schneider, A., et al. 2016, ApJ, 224, 36

Kirkpatrick, J. D., Reid, I. N., Liebert, J., et al. 2000, AJ, 120, 447

Kirkpatrick, J. D., Schneider, A., Fajardo-Acosta, S., et al. 2014, ApJ, 783, 122

Kowalski, A. F., Hawley, S. L., Holtzman, J. A., Wisniewski, J. P., \& Hilton, E. J. 2010, ApJL, 714, L98

Kowalski, P. M., \& Saumon, D. 2006, ApJ, 651, 137

Lépine, S. 2008, AJ, 135, 2177

Lépine, S., Rich, M., \& Shara, M. 2003, AJ, 125, 1598

Lépine, S., Rich, M., \& Shara, M. 2007, ApJ, 669, 1235

Lépine, S., \& Shara, M. M. 2005, AJ, 129, 1483

Luyten, W. J. 1976, A Catalogue of Stars with Proper Motions Exceeding 0.5" Annually (Minneapolis, MN: Univ. Minnesota Press)

Mermilliod, J.-C., Mermilliod, M., \& Hauck, B. 1997, A\&AS, 124, 349

Oke, J. B., Cohen, J. G., Carr, M., et al. 1995, PASP, 107, 375

Oke, J. B., \& Gunn, J. E. 1982, PASP, 94, 586

Oppenheimer, B. R., Hambly, N. C., Digby, A. P., Hodgkin, S. T., \& Saumon, D. 2001, Sci, 292, 698

Pecaut, M. J., \& Mamajek, E. E. 2013, ApJS, 208, 9

Rayner, J. T., Cushing, M. C., \& Vacca, W. D. 2009, ApJS, 185, 289

Rayner, J. T., Toomey, D. W., Onaka, P. M., et al. 2003, PASP, 115, 362

Reid, I. N., \& Hawley, S. L. 2005, New Light on Dark Stars, Red Dwarfs, Low-Mass Stars, Brown Dwarfs (Chichester: Springer-Praxis)

Reid, I. N., Kirkpatrick, J. D., Gizis, J. E., et al. 2000, AJ, 119, 369

Reid, N. 1997, in ASP Conf. Ser. 127, In Proper Motions and Galactic Astronomy, ed. R. M. Humphreys (San Francisco, CA: ASP), 63

Ryan, S. G., \& Norris, J. E. 1991, AJ, 101, 1835

Salim, S., \& Gould, A. 2003, ApJ, 582, 1011

Salim, S., Rich, R. M., Hansen, B. M., et al. 2004, ApJ, 601, 1075

Saumon, D., Bergeron, P., Lunine, J. I., Hubbard, W. B., \& Burrows, A. 1994 ApJ, 424, 333

Savcheva, A. S., West, A. A., \& Bochanski, J. J. 2014, ApJ, 794, 145

Scholz, R.-D., Storm, J., Knapp, G. R., \& Zinnecker, H. 2009, A\&A, 494, 949

Skrutskie, M. F. 1997, in The Impact of Large Scale Near-IR Sky Surveys, ed F. Garzon et al. (Dordrecht: Kluwer), 25

Tremblay, P. E., \& Bergeron, P. 2008, ApJ, 672, 1144

Vacca, W. D., Cushing, M. C., \& Rayner, J. T. 2003, PASP, 115, 389

Wright, E. L., Eisenhardt, P. R. M., Mainzer, A. K., et al. 2010, AJ, 140, 1868

York, D. G., Adelman, J., Anderson, J. E., et al. 2000, AJ, 120, 1579 